\title{
CONTINUOUS COLLECTIONS OF CONTINUOUS CURVES IN THE PLANE
}

\author{
R. D. ANDERSON ${ }^{1}$
}

The purpose of this paper is to consider the class of continuous collections of mutually exclusive compact continuous curves in the plane. Throughout this paper we shall denote by $G$ or $G$ with a subscript or superscript a collection of this class with the property that $G$ with respect to its elements as points is a nondegenerate compact closed point set. $G$ has then the significance of being both a collection of continua and a point set itself.

By a continuous curve will be meant a nondegenerate locally connected compact continuum. By a continuous collection will be meant a collection which is both upper and lower semi-continuous. By a (simple) chain will be meant a finite collection $x_{1}, x_{2}, \cdots, x_{n}$ of open discs (i.e. interiors of simple closed curves) such that $\bar{x}_{i} \cdot \bar{x}_{j}$ exists if and only if $|i-j| \leqq 1$ and is the closure of an open disc (i.e. a 2-cell) if it does exist. The $x_{i}$ are called links of the chain. A subchain of a chain $c$ is a chain whose links are links of $c$.

A chain $c$ will be said to simply cover a set $M$ if $c^{*}$ contains $M$ and if for no proper subchain $c^{\prime}$ of $c$ does the closure of $c^{\prime *}$ contain $M$. Two chains will be said to be mutually exclusive if no link of either intersects any link of the other. A collection $C^{\prime}$ of sets is said to be a (closed) refinement of a collection $C$ of sets if (the closure of) each element of $C^{\prime}$ is a subset of some element of $C$. An emanation point of a continuum $M$ is a point which is the common part of each pair of some three nondegenerate subcontinua of $M$. A hereditary continuous curve is a continuous curve each of whose nondegenerate subcontinua is a continuous curve.

It is immediately clear that if $G$ is connected, $G$ contains uncountably many elements and that only countably many can contain triods [1]. Except for a countable number of elements, each element of $G$ must be either an arc or a simple closed curve. We denote the elements of $G$ which are neither arcs nor simple closed curves by $g_{1}, g_{2}, g_{3}, \cdots$. From the hypothesis of continuity of $G$ it follows immediately that no element of a connected $G$ contains a 2-cell.

THEOREM I. It is not true that both the collection of arcs and the collection of simple closed curves are dense in $G$.

Presented to the Society, December 26,1951; received by the editors December 1, 1951.

${ }^{1}$ Research sponsored by the Office of Naval Research. 
Proof. We suppose the contrary. Then there exists an arc $t_{1}$, a simple chain $c_{1}$ which simply covers $t_{1}$ and is of at least eleven links and of link diameter less than one, and two open sets $d_{1}$ and $d_{2}$ containing the end points of $t_{1}$ such that the $d_{i}$ lie in distinct end links of $c_{1}$ and intersect no other links of $c_{1}$. We consider the set $F$ of all elements of $G$ lying in $c_{1}^{*}$ and intersecting $d_{1}$ and $d_{2} . F$ is open and contains a simple closed curve $j_{1}$ as an element. But $j_{1}$ contains two mutually exclusive arcs each intersecting $d_{1}$ and $d_{2}$ and hence every link of $c_{1}$. Some arc $t_{2}$ of $F$ must then contain two mutually exclusive subarcs each intersecting every link of $c_{1}$, as the collection $G$ is continuous, the collection of arcs of $G$ is dense in $G$, and there exist two mutually exclusive simple chains each a closed refinement of $c_{1}$, the sum of the links of each of which intersects every link of $c_{1}$ such that, for some open subset $F_{1}$ of $F$ containing $j_{1}$, every element of $F_{1}$ must intersect each link of both chains. By an iteration of the above argument it follows that there exists a sequence of chains $c_{1}, c_{2}, \cdots$ such that for each $i, c_{i+1}$ is a closed refinement of $c_{i}, c_{i}$ is of link diameter less than $1 / i, c_{i}^{*}$ contains an $\operatorname{arc} t_{i}$ of $G$ intersecting each link of $c_{i}, c_{i}^{*}$ does not contain $g_{i}$, and $c_{i+1}$ contains two mutually exclusive simple subchains each of which intersects every link of $c_{i}$. $H=c_{1}^{*} \cdot c_{2}^{*} \cdot c_{3}^{*} \cdots$ cannot be an arc or a simple closed curve for $H$ contains uncountably many mutually exclusive continua each of diameter greater than some number. As $H$ is the sequential limiting set of the sequence of elements $t_{i}$ of $G, H$ must be an element of $G$, and as $H$ cannot be $g_{i}$ for any $i$, it must be an arc or a simple closed curve. Thus Theorem I is established. We note here also that a simple argument could be given to show that $H$ is not even locally connected.

We designate by $\alpha$ either the point at infinity $\omega$ or any particular point of the plane.

Lemma A. If $f_{1}, f_{2}, f_{3}, \cdots$ is a sequence $V$ of elements of a collection $G$ such that, for each $i, f_{i+1}$ separates $f_{i}$ from $\alpha$ in the plane, then $V$ has a sequential limiting set $k$ which is an element of $G$ and which either contains $\alpha$ or, for each $i$, separates $f_{i}$ from $\alpha$.

Proof. We note that for $i>j, f_{i}$ separates $f_{j}$ from $\alpha$. Some subsequence $V^{\prime}$ of $V$ has a sequential limiting set $k^{\prime}$ which from the asasumption of continuity of the collection $G$ is an element of $G$. Let $k^{\prime \prime}$ be the sequential limiting set of some other subsequence $V^{\prime \prime}$ of $V$. We wish to show that $k^{\prime}=k^{\prime \prime}$ and therefore that $k^{\prime}$ is the sequential limiting set of $V$ itself. For any element $f_{n_{j}}$ of $V^{\prime}, k^{\prime}$ either contains $\alpha$ or separates $f_{n_{j}}$ from $\alpha$, for $k^{\prime}$ is the common part of a monotonic se- 
quence of closed and compact sets each either containing $\alpha$ or separating $f_{n_{j}}$ from $\alpha$. If some point $P$ of $k^{\prime}$ is not a limit point of the sum of the elements of $V^{\prime \prime}$, then it readily follows that, for some $i>j, f_{i}$ does not separate $f_{j}$ from $\alpha$ as no point of $k^{\prime}$ is separated from $\alpha$ by any element of $V$. It follows that $k^{\prime}$ is the sequential limiting set $k^{\prime \prime}$ of $V^{\prime \prime}$ as $V^{\prime \prime}$ has by hypothesis a sequential limiting set. By the argument suggested above for $V^{\prime}, k\left(=k^{\prime}=k^{\prime \prime}\right)$ must contain $\alpha$ or separate each element of $V$ from $\alpha$.

Lemma B. If $g$ an element of $G$ is a limit element of each of two subsets $G_{1}$ and $G_{2}$ of $G$, with $G_{1}^{*}$ and $G_{2}^{*}$ lying in distinct complementary domains of $g$ in the plane, then $g$ is a simple closed curve.

Proof. This lemma follows immediately from the continuity of the collection $G$, the elementary separation properties of a simple closed curve in the plane, and Theorem ${ }^{2} 41$ on p. 216 of reference [2].

Lемма C. If in addition to the hypotheses of Lemma A it is also supposed that $G$ is connected and $k$ does not contain $\alpha$, then any nondegenerate subcontinuum $G^{\prime}$ of $G$ which contains $k$ and contains no element of $G$ not separated by $k$ from $\alpha$ must contain some term of $V$.

Proof. It follows from Lemma B that $G^{*}-k$ and $f_{1}+f_{2}+\cdots$ all lie in the same complementary domain of $k$. But then there exists an arc $s$ in such complementary domain of $k$ from a point of $G^{*}-k$ to some point of some $f_{i}$ such that $s$ does not intersect any $f_{j}, j>i$. Otherwise $s$ must intersect $k$. But then $f_{i+1}$ does not separate $f_{i}$ from $\alpha$, contrary to hypothesis.

Leмma D. If $G$ does not contain any arcs as elements and is connected, then $G$ does not contain two elements such that neither separates the other from $\omega$ in the plane.

Proof. Suppose the contrary and let $A$ and $B$ be such elements. There is a continuum $G^{\prime}$ in $G$ which contains $A$ and $B$ and is irreducible with respect to being a continuum and containing $A$ and $B$. From Lemmas $\mathrm{A}$ and $\mathrm{B}$ or $\mathrm{C}$ with a straightforward argument it follows that if there is any element of $G^{\prime}$ separating $A$ from $\omega$ and not separating $B$ from $\omega$, there is an element $A^{\prime}$ doing this such that no element of $G^{\prime}$ separates $A^{\prime}$ from $\omega$ and does not separate $B$ from $\omega$. We note that $B$ is not $A^{\prime}$ and we denote by $A^{\prime \prime}$ the set $A$ if no $A^{\prime}$ exists or $A^{\prime}$ if such does exist. Similarly we define a set $B^{\prime \prime}$ with respect to $A^{\prime \prime}$ and $\omega$. But as $G^{\prime}$ contains $A^{\prime \prime}$ and $B^{\prime \prime}$ and is irreducible with

${ }^{2}$ If $D$ and $E$ are two complementary domains of a compact continuous curve $M$, the outer boundary of $D$ with respect to $E$ is a simple closed curve. 
respect to being a continuum and containing $A$ and $B$, it follows that no element of $G^{\prime}$ not separated by $A^{\prime \prime}$ from $B$ and $\omega$ and not separated by $B^{\prime \prime}$ from $A^{\prime \prime}$ and $\omega$ can separate the plane into two complementary domains each of which contains an element of $G^{\prime}$ such that $A^{\prime \prime}$ and $B^{\prime \prime}$ are in the same complementary domain. But then as $G^{\prime}$ is connected there must be uncountably many mutually exclusive open sets in the plane, which is the desired contradiction.

Theorem II. ${ }^{3}$ If $G$ is connected and every element of $G$ is a simple closed curve, then $G$ is an arc and $G^{*}$ is an annulus whose boundary simple closed curves are the end elements of $G$.

Proof. From Lemma D we can conclude that of every three elements of $G$ one separates the other two from each other and hence $G$ is an arc. The end elements of $G$ do not separate $G$ and are simple closed curves. One separates the other from $\omega$. Hence they bound an annulus $A$ which contains the other elements of $G$. From the elementary separation properties of simple closed curves, Lemma A, and the continuity of $G$, it readily follows that $G^{*}$ fills up $A$.

THEOREM III. If $G$ does not contain any arcs as elements and is connected, then $G^{*}$ contains a 2-cell.

Proof. From Lemma D we conclude again as above that $G$ is an arc. From Lemma B it follows that every cut element of $G$ is a simple closed curve. Hence for a subarc $G^{\prime}$ of $G$ each element of $G^{\prime}$ is a simple closed curve and Theorem II implies that $G^{*}$ and hence $G^{*}$ contains a 2-cell.

Definition. A sequence $c_{1}, c_{2}, c_{3}, \cdots$ of simple chains will be said to be hooked provided that $c_{1}$ contains an odd number $(>7)$ of links and there exists a point $P$ in the middle link of $c_{1}$ and sequences $d_{2}^{\prime}, d_{3}^{\prime}, d_{4}^{\prime}, \cdots$ and $d_{2}^{\prime \prime}, d_{3}^{\prime \prime}, d_{4}^{\prime \prime}, \cdots$ of simple chains such that for each $i,(1) c_{i+1}$ is a closed refinement of $c_{i}$ and $d_{i+1}^{\prime}$ and $d_{i+1}^{\prime \prime}$ are closed refinements of $d_{i}^{\prime}$, (2) $d_{i+1}^{\prime}$ and $d_{i+1}^{\prime \prime}$ are mutually exclusive subchains of $c_{i+1}$, (3) exactly one link of $d_{i+1}^{\prime}$ contains $P$, (4) the end links of $d_{2}^{\prime}$ are in the end links of $c_{1}$, (5) the end links of $d_{i+1}^{\prime}$ are in the end links of $d_{i}^{\prime},(6) d_{2}^{\prime \prime}$ contains a link in the link of $c_{1}$ containing $P$ and a link in one end link of $c_{1}$, and (7) $d_{i+1}^{\prime \prime}$ contains a link in the link of $d_{i}^{\prime}$ containing $P$ and a link in one end link of $d_{i}^{\prime}$.

An $\epsilon$-sequence of chains is a sequence of chains $c_{1}, c_{2}, c_{3}, \cdots$ with the property that if $\epsilon$ is any positive number, there exists a number $N_{\epsilon}$ such that for $j>N_{\epsilon}$, each link of $c_{j}$ is of diameter less than $\epsilon$.

\footnotetext{
Theorem II is a special case of Theorem 4 and Lemma 4.3 of reference [6].
} 
LEMma E. If $M$ is a continuum which is simply covered by each chain of a hooked $\epsilon$-sequence of chains, then $M$ is not locally connected.

Proof. That Lemma $E$ is true follows from the observation that every point $P$ of a continuum $M^{\prime}$ in the intersection of the $d_{i}^{\prime}$ is a limit point of the sum of continua $M_{i}$ one in each set $d_{i}^{\prime \prime}$ and that every sufficiently small open set containing $P$ has infinitely many components with no two points of distinct $M_{i}$ in the same component.

THEOREM IV. If $G$ is connected and contains no simple closed curve as an element, then $G^{*}$ contains a 2-cell.

Proof. Let $c_{1}$ be a simple chain of an odd number $(>7)$ of links simply covering an arc $t_{1}$ which is an element of $G$ and let $d_{1}$ and $d_{2}$ be connected open sets one in each of the end links of $c_{1}$ such that $\bar{d}_{1}+\bar{d}_{2}$ intersects the closure of no cut link of $c_{1}$ and each $d_{i}(i=1,2)$ contains an end point of $t_{1}$. From the continuity of the collection it follows that there exists a connected open set $E$ containing $t_{1}$ and contained in $c_{1}^{*}$ such that every element of $G$ intersecting $E$ lies in $E$ and intersects $d_{1}$ and $d_{2}$ (and hence every link of $c_{1}$ ). Let $G_{1}$ be a subcontinuum of $G$ containing $t_{1}$ with $G_{1}^{*}$ in $E$. Let $m$ be the middle link of $c_{1}$ and suppose that $m \cdot G_{1}^{*}$ contains no 2-cell. Let $s_{1}$ and $s_{2}$ be arcs which lie in the closures of the end links of $c_{1}$ each intersecting the boundary of $c_{1}$ exactly in its end points such that for each $i(i=1,2) s_{i}$ separates $d_{i}$ from $m$ in the closure of $c_{1}^{*}$ and intersects the closure of only one link of $c_{1}$ and such that $s_{1}$ and $s_{2}$ form together with two arcs $z_{1}$ and $z_{2}$ of the boundary of $c_{1}^{*}$ a simple closed curve $J$ whose interior contains all non-end links of $c_{1}$ and whose exterior contains $d_{1}+d_{2}$. Let $H$ be a maximal set of mutually exclusive arcs such that each has an end point and only an end point in common with each $s_{i}$ and each is a subset of an element of $G_{1}$. Each element of $G_{1}$ contains at least one arc in $H$. Each arc $h$ of $H$ separates $I=J+\operatorname{Int}(J)$ into two mutually separated connected sets and on the basis of this separation property the elements of $H$ form a totally ordered set.

The supposition that $m \cdot G_{1}^{*}$ contains no 2 -cell implies that there exists a connected open set $D$ in $m$ with the property that $H$ is the sum of two uncountable mutually exclusive sets $H_{1}$ and $H_{2}$ such that $D$ is separated in $I$ from $z_{i}$ by every element of $H_{i}$. We assume now that no element of $G_{1}$ contains two mutually exclusive elements of $H$. As $G_{1}$ is connected and continuous, some point $Q$ must be a limit point of each of the sets $H_{1}^{*}$ and $H_{2}^{*}$. Then as follows from sequential limiting properties there exist continua $K_{1}$ and $K_{2}$ each containing $Q$ 
and each intersecting $s_{1}$ and $s_{2}$, with $K_{1}+K_{2}$ contained in an element $f$ of $G_{1}$ and with $K_{i}$ separating $D$ in $I$ from each element of $H_{i}$ not in $K_{i}$. Let $K^{\prime}$ be the component of $f \cdot I$ which contains $K_{1}+K_{2} . K^{\prime}$ is locally connected and contains an arc $r$ intersecting each set $s_{i}$ in exactly an end point of $r . K^{\prime}+s_{1}+s_{2}$ separates $D$ in the plane from all elements of $H_{1}$ or $H_{2}$ not in $K^{\prime}$. Let $H_{i}^{\prime}$ denote the set of all elements in $H_{i}$ not in $K^{\prime} . H_{1}^{\prime}{ }^{*}$ and $H_{2}^{\prime}{ }^{*}$ are mutually separated and together with the set of elements of $H$ in $K^{\prime}, H_{1}^{\prime}$ and $H_{2}^{\prime}$ contain all elements of $H$. By Theorem 41 on p. 216 of reference [2] quoted above the outer boundary with respect to $\omega$ of the complementary domain of $K^{\prime}+s_{1}+s_{2}$ containing $D$ is a simple closed curve $Y$. There exists an arc $r^{\prime}$ of $K^{\prime}$ such that $r^{\prime}$ lies in $I$, has exactly an end point on each set $s_{i}$, and separates $H_{1}^{\prime}{ }^{*}$ from $H_{2}^{\prime}{ }^{*}$ in $I$, and such that $K^{\prime}$ contains a point $Q$ in $m$ not in $r^{\prime}$. That such $r^{\prime}$ exists may be seen by considering $Y$ and $r$. If we let $P_{1}$ and $P_{2}$ be the points of $Y$ on $r$ closest on $r$ to $s_{1}$ and $s_{2}$ respectively and note that $Y$ is the sum of two arcs $y_{1}$ and $y_{2}$ from $P_{1}$ to $P_{2}$ having just $P_{1}+P_{2}$ in common, at least one of the sets $y_{1}$ and $y_{2}$ (say $y_{1}$ ) contains a point of $m$. Let $r^{\prime}$ be an arc which is contained in $r+y_{2}$, intersects $y_{1}$ in at most $P_{1}$ and $P_{2}$ and intersects each of the sets $s_{1}$ and $s_{2}$ in exactly an end point of itself.

As $G_{1}$ is connected, $f$ must be a limit element of each of the two subsets of $G_{1}$ whose elements contain elements of $H_{1}^{\prime}$ and $H_{2}^{\prime}$ respectively. But then as the set of arcs of $G_{1}$ is dense in $G_{1}$, there must be an arc $t_{2}$ of $G_{1}$ which contains two mutually exclusive subarcs, one in $H$ and the other interesecting $m$ and either $s_{1}$ or $s_{2}$. We also note that the existence of such an arc $t_{2}$ immediately follows if we assume that some element of $G_{1}$ contains two elements of $H$. There exists a chain simply covering $t_{2}$ and of diameter less than $1 / 2$ which satisfies all conditions for the second chain of a hooked sequence of chains whose first chain is $c_{1}$ where the point $P$ of the definition could be any point of $m \cdot d_{2}{ }^{\prime}$. By an iteration of the above argument without essential modification it can be established that there exists a hooked $\epsilon$-sequence of simple chains $c_{i}$ each term of which simply covers an element of $G$. But the common part of the $c_{i}$ is not locally connected by Lemma $\mathrm{E}$ and is locally connected as an element of $G$ by hypothesis. Therefore $G^{*}$ contains a 2 -cell.

THEOREM V. If $G$ is connected, $G^{*}$ contains a 2-cell.

Proof. Theorem V follows immediately from Theorems I, III, and IV.

Theorem VI. If $G$ is connected, then $G$ with respect to its elements as 
points is a hereditary continuous curve such that the closure of the set of emanation points of $G$ is totally disconnected.

Proof. Suppose some subcontinuum $G^{\prime}$ of $G$ is not locally connected at some point $P$. Then there must be a subcontinuum $G^{\prime \prime}$ of $G^{\prime}$ such that $G^{\prime}$ is not locally connected at any point of $G^{\prime \prime}$ and such that each point of $G^{\prime \prime}$ is a limit point of $G^{\prime}-G^{\prime \prime}$. By Theorem V, $G^{\prime \prime *}$ must contain a 2-cell $K$. But as the collection $G^{\prime}$ is continuous and hence as no element of $G^{\prime \prime}$ containing an interior point of $K$ can be a limit element of $G^{\prime}-G^{\prime \prime}$, we have a contradiction. We therefore conclude that $G$ is a hereditary continuous curve. Suppose now that the closure of the set $H$ of emanation points of $G$ is not totally disconnected and let $H^{\prime}$ be an arc in $\bar{H}$. But as above $H^{\prime *}$ contains a 2-cell and every point in $H^{\prime}$ is a limit point of $G-H^{\prime}$ which implies a contradiction similar to that above. Thus Theorem VI is established.

It should be noted that Theorem VI gives necessary but not sufficient conditions which the continuum $G$ must satisfy. Sufficiency conditions would seem to be considerably more complicated. Presumably they would involve among other things use of Theorems I and VI and Lemma C in conjunction with the well known theorem of reference [2] that the continuous monotone image of a sphere is a cactoid. Theorem VII of this paper establishes that if $G$ is restricted to being a subset of a plane, then the conditions of Theorem VI are sufficient in this special case.

We now consider some examples of collections $G$ in which $G$ is an arc and the elements of $G$ are arcs.

EXAMPLE 1. If $M$ is the unit square in the plane, the collection $G$ of vertical line intervals of length 1 in $M$ is a continuous collection of mutually exclusive arcs filling up $M$.

EXAmple 2. If $M$ is the unit square in the plane, there exists a collection $G^{\prime}$ of mutually exclusive arcs filling up $M$ in which the intervals $[(0,1 / 2),(1 / 4,1 / 2)]$ and $[(3 / 4,1 / 2),(1,1 / 2)]$ are elements of $G^{\prime}$ and each other element of $G^{\prime}$ has both end points on the boundary of $M$, one with ordinate greater than $1 / 2$ and the other with ordinate less than $1 / 2$.

Example 3. Let $M$ be the set consisting of the interval I: $[(0,-1)$, $(0,1)]$, the interval $I^{\prime}:[(2,-1),(2,1)]$, the curves

$$
y_{1}=\left\{\begin{array}{c}
\sin \frac{\pi}{x}, 0<x \leqq 1 \\
-\sin \frac{\pi}{2-x}, 1<x<2
\end{array}\right.
$$




$$
y_{2}=\left\{\begin{array}{l}
x / 10+\sin \frac{\pi}{x}, 0<x \leqq 1, \\
(2-x) / 10-\sin \frac{\pi}{2-x}, 1<x<2,
\end{array}\right.
$$

and those points on vertical line segments with end points in $y_{1}$ and $y_{2}$. Let $G$ be a collection filling up $M$ which consists of $I, I^{\prime}$, and mutually exclusive arcs each of which lies in $M$ and has exactly an end point on $y_{1}$ and exactly an end point on $y_{2}$ (and hence separates $M$ ) with the $x$-coordinate $x_{1}$ of the end point on $y_{1}$ and the $x$-coordinate $x_{2}$ of the end point on $y_{2}$ satisfying the relationship $x_{2}-x_{1}=2 x_{1} x_{2}$ whenever $x_{1}$ and $x_{2}$ are both less than $1 / 2$ and $x_{2}-x_{1}=2\left(2-x_{1}\right)\left(2-x_{2}\right)$ whenever $x_{1}$ and $x_{2}$ are both greater than $3 / 2$. It is clear that such a continuous collection can exist and will be an arc.

ExAmple 4. Let $M^{\prime}$ be the set $M$ of Example 3 plus those points with $0 \leqq x \leqq 2$ and $y_{3}<y \leqq 2$ with $y_{3}(x)$ the maximal ordinate of a point in $M$ for particular $x$. Let the collection consist of all arcs each of which is an arc of $G$ in Example 3 and a vertical interval from $M$ to the line $y=2$.

It is not difficult to construct an example which is essentially a modification of Examples 2 and 4 such that the collection $G$ of mutually exclusive arcs is again itself an arc, the set of points in the boundary of $M$ which are accessible from $S-M$ is totally disconnected, and no local cross section of $G$ exists. We also note that if $N$ in the plane is the Cartesian product of a Cantor discontinuum and an interval, $j$ is an integer, and $u$ and $v$ are components of $N$, there exist $j$ sets of the type of Example 3 with $u$ and $v$ as end elements, each of the $j$ sets intersecting $N$ or any other of the $j$ sets in exactly $u+v$.

THEOREM VII. If $J$ is a plane hereditary continuous curve such that the closure of the set of emanation points of $J$ is totally disconnected, there exist a continuum $M$ in the plane and a continuous collection $G$ of arcs filling up $M$ such that $G$ with respect to its elements as points is homeomorphic to $J$.

Proof. Let $H$ be the set of all emanation points of $J$ and $E$ the set of all end points of $J . \bar{H}+E$ is a closed and totally disconnected point set, for if $P$ not in $\bar{H}$ is a limit point of $E$, then it follows directly that $J$ is not locally connected at $P$ and as this observation also implies that $E$ contains at most a countable number of points not in $\bar{H}$, it follows that $\bar{H}+E$ is totally disconnected. $J-(\bar{H}+E)$ is the sum of a countable collection $Q$ of mutually exclusive connected sets open 
relative to $J$ each a subset of an arc having both end points in $\bar{H}+E$. For any $\epsilon>0$ not more than a finite number of elements of $Q$ are of diameter greater than $\epsilon$ (as $J$ is a hereditary continuous curve).

By [3] there exists in the plane an arc $t$ containing $\bar{H}+E$. It is possible that $t$ may intersect $J$ in points other than $\bar{H}+E$ and in fact may have nondegenerate continua in common with $J$. Let $V^{\prime}$ be an open curve containing $t$. There exists an open curve $V$ containing $\bar{H}+E$ such that $V \cdot J$ is totally disconnected. That such a $V$ exists may be easily demonstrated by considering a slight modification of $V^{\prime}$. Let $V \cdot J=N$. There exists a homeomorphism of the plane onto itself carrying $V$ onto the $x$-axis, $J$ onto a set $J^{\prime}$, and $N$ onto a set $N^{\prime}$ on the interval $[(0,0),(1,0)] . J^{\prime}$ is the sum of $N^{\prime}$ and a countable collection $R$ of arcs $k_{1}, k_{2}, k_{3}, \cdots$ each having its end points in $N^{\prime}$ and having no other points on the $x$-axis. For any $\epsilon>0$ at most a finite number of the elements of $R$ are of diameter greater than $\epsilon$ and no point not in $N^{\prime}$ is common to two elements of $R$. Let $Z$ be the set of all vertical intervals of length one with midpoints on $N^{\prime}$. M will consist of $Z+\sum_{i=1}^{\infty} M_{i}$ where, for each $i, M_{i}$ is a set to be defined in terms of $\sum_{j=1}^{i-1} M_{j}$ and the set $k_{i}$.

$M_{1}$ is to be a set of the type of Example 3 whose end elements are the elements of $Z$ containing the end points of $k_{1} . M_{1}$ is to contain no points with $y<-1 / 2$ if $k_{1}$ lies (except for its end points) above the $x$-axis or no points with $y>1 / 2$ if $k_{1}$ lies (except for its end points) below the $x$-axis. $M_{1}$ is to contain no point with $|y|>1 / 2+$ the distance between the end points of $k_{1}, M_{1} \cdot Z$ is to be exactly the two elements of $Z$ containing the end points of $k_{1}$, and the projection of $M_{1}$ on the $x$-axis is to be the interval whose end points are the end points of $k_{1}$. It is clear that such a set $M_{1}$ can exist and from the definition of $M_{1}$ that there exists a set $G_{1}$ of arcs filling up $M_{1}$ such that $G_{1}$ is an arc whose end elements are in $Z$. If $\sum_{j=1}^{i-1} M_{j}$ is defined, then $M_{i}$ and $G_{i}$ can be defined similarly from $k_{i}$ as were $M_{1}$ and $G_{1}$ from $k_{1}$ with the added condition that $M_{i}$ does not intersect $\sum_{j=1}^{i-1} M_{j}$. That such an $M_{i}$ always exists follows in part from the fact that, for $i \neq j, k_{i}$ does not intersect $k_{j}$ in a cut point of either, and that for any $i, k_{i}$ lies except for its end points entirely on one side of the $x$-axis. The set $G$ is the set $Z+\sum_{i=1}^{\infty} G_{i}$. It is clear from the construction outlined above that $M$ is a compact continuum and that $G$ is homeomorphic with $J$.

Theorem ${ }^{4}$ VIII. If $M$ is a 2-cell and $G$ fills up $M$, then $G$ is an arc.

Proof. $G$ must be a dendron, for $G$ is a hereditary continuous curve

${ }^{4} \mathrm{G}$. S. Young has announced a result [5] which implies Theorems VIII and IX. 
by Theorem VI and the collection $F$ consisting of the elements of $G$ and the individual points of $S-M$ (where $S$ is a 2-sphere containing $M$ ) must by (4) be a cactoid $\Sigma$. But the elements of $F$ not in $G$ form an open disc in $\Sigma$, and if $G$ contains a simple closed curve it must contain an interior of such simple closed curve in a sphere of $\Sigma$ and hence $G$ must not contain a simple closed curve. If $G$ contains a branch point $g$, then $g$ separates $G^{*}$ in to at least three complementary domains and it follows from Lemma $B$ that at least two, say $D_{1}$ and $D_{2}$, must contain points of the boundary of $M$. But then some arc $t$ of $g$ with exactly its end points on the boundary of $M$ must separate $D_{1}$ and $D_{2}$. Then $g$ must be $t$ from the continuity of $G$ and separation properties of arcs and simple closed curves and hence $g$ is not a branch point. Finally, as a dendron without branch points is an arc, Theorem VIII is established.

THEOREM IX. If $F$ is a continuous collection of mutually exclusive locally connected compact continua filling up the plane $S$ and there exist two elements of $F$ which do not separate $S$, then all elements of $F$ are degenerate.

Proof. It is clear from the continuity of $F$ that if $F$ contains a nondegenerate element, the set $E$ of all nondegenerate elements of $F$ is open in $F$. We assume that $E$ exists. If $F^{\prime}$ is the one point compactification of $F$ then, by [4], $F^{\prime}$ is a cactoid. If $F^{\prime}$ contains a 2 -sphere $U$, then some element of $F^{\prime}$ in $U$ is also in $E$ and as $E$ is open in $F^{\prime}$ there exists a 2-cell in $U$ each of whose points is an element of $E$. By Theorem VI this is impossible and hence $F^{\prime}$ is a dendron. But $F^{\prime}$ by hypothesis contains at least three elements not separating $F^{\prime}$ (one the point in $F^{\prime}$ not in $F$ ) and hence $F^{\prime}$ contains a branch point $P$, but from Lemma B, $P$ must be a simple closed curve (for it must separate the plane as $F$ fills up the plane) and then from elementary separation properties of simple closed curves $P$ cannot be a branch point of $F^{\prime}$. Thus the set $E$ cannot exist under the hypotheses of the theorem and Theorem IX is established.

THEOREM X. If $F$ is a continuous collection of mutually exclusive compact locally connected continua filling up the plane $S$, then either $G$ is the collection of all points of $S$ or $G$ is a ray the end element of which does not contain a simple closed curve (and may be degenerate) and all other elements of which are simple closed curves.

Proof. Theorem $\mathrm{X}$ can be readily established from Theorem IX and Lemma B. 


\section{REFERENCES}

1. R. L. Moore, Concerning triods in the plane and the junction points of plane continua, Proc. Nat. Acad. Sci. U.S.A. vol. 14 (1928) pp. 85-88.

2. - Foundations of point set theory, Amer. Math. Soc. Colloquium Publications, vol. 13, 1932.

3. R. L. Moore and J. R. Kline, On the most general plane closed point set through which it is possible to pass a simple continuous arc, Ann. of Math. vol. 20 (1919) pp. 218-223.

4. R. L. Moore, Concerning upper semi-continuous collections of continua, Trans. Amer. Math. Soc. vol. 27 (1925) pp. 416-428.

5. G. S. Young, Interior and border transformations on surfaces, Bull. Amer. Math. Soc. Abstract 52-5-220.

6. Herman Cohen, Some results concerning homogeneous plane continua, Duke Math. J. vol. 18 (1951) pp. 467-474.

The Institute for Advanced Study and

The University of Pennsylvania

\section{DIMENSION AND DISCONNECTION}

\section{BYRON H. MCCANDLESS}

Let $X$ be a semi-compact separable metric space. We shall prove the following theorem using results found in Hurewicz and Wallman's book Dimension theory (Princeton University Press, 1948):

THEOREM. $\operatorname{dim} X \leqq n$ if and only if any closed subset of $X$ containing at least two points can be disconnected by a closed set of dimension $\leqq n-1$.

The necessary and sufficient condition stated in the theorem was found in looking for an $n$-dimensional analogue of the property of a space being totally disconnected (property $\alpha_{0}$ below) and will be denoted by $\alpha_{n}$.

Hurewicz and Wallman show (p. 20) that the following three properties of the space $X$ are equivalent:

$\alpha_{0} . X$ is totally disconnected.

$\beta_{0}$. Any two points in $X$ can be separated.

$\gamma_{0}$. Any point can be separated from a closed set not containing it, that is, $\operatorname{dim} X=0$.

They also show (p. 36) that the following $n$-dimensional analogues of $\beta_{0}$ and $\gamma_{0}$ are equivalent:

$\beta_{n}$. Any two points in $X$ can be separated by a closed set of dimension $\leqq n-1$.

Received by the editors December 5, 1951. 